\title{
Low-force contact heating and softening using micromechanical switches in diffusive-ballistic electron-transport transition
}

\author{
Brian D. Jensen, Kuangwei Huang, Linda L.-W. Chow, and Katsuo Kurabayashi ${ }^{\text {a) }}$ \\ Department of Mechanical Engineering, University of Michigan, Ann Arbor, Michigan 48109
}

(Received 5 May 2004; accepted 11 November 2004; published online 5 January 2005)

\begin{abstract}
We demonstrate softening of the gold-to-gold contact in surface micromachined microelectromechanical switches under electrostatic force near $30 \mu \mathrm{N}$, which results from the heating of contact asperities sustaining electron transport. A bias potential that causes the switch contacts to soften is measured for initial contact resistance varying between 0.5 and $300 \Omega$. The asperity sizes in this range are comparable to the electron mean-free path at room temperature. We show that contact spots smaller than the mean-free path require larger bias for softening. Our results can be explained using a model accounting for ballistic electron transport in the contact. () 2005 American Institute of Physics. [DOI: 10.1063/1.1850191]
\end{abstract}

Microelectromechanical systems (MEMS) devices often allow the investigation of nanometer-scale phenomena that are difficult to study using macroscopic instruments. This paper describes experiments performed using MEMS switches to study the heating of metal contacts with size in the transition region between ballistic and diffusive electron transport. The contact radius of these contacts is nearly the same as the mean-free path for electron-phonon scattering. Such small contacts have previously been created using plastic deformation of the aluminum oxide layer in aluminum contacts $^{1}$ or by manipulating thin wires using piezoelectric actuators. ${ }^{2}$ Small-area contacts have also been generated using an atomic force microscope $e^{3}$ as well as an interfacial force microscope. ${ }^{4}$ However, none of these references has studied the heating of small contact spots due to the passage of current.

Several authors (such as Ref. 5) have developed models of contact heating for diffusive contacts, deriving the wellknown voltage-temperature relation

$$
V^{2}=8 \int_{T_{0}}^{T_{a}} \rho \kappa d T
$$

in which $V$ is the voltage drop in the contact spot, $T_{0}$ is the temperature of the contact bodies, $T_{a}$ is the temperature of the contact spot, $\rho$ is the electrical resistivity of the material, and $\kappa$ is its thermal conductivity. However, for contact spots whose size is on the order of the electron mean-free path, this relation no longer holds. ${ }^{1}$ However, no clear presentation of a revised model for small contact spots has been suggested, though contact heating of ballistic-scale contacts has been experimentally demonstrated. ${ }^{6}$

This paper shows evidence of heating in small contacts by using the contact softening phenomenon. ${ }^{5}$ As the contact temperature rises, a threshold temperature is reached which allows diffusion of dislocations away from the contact spot, decreasing surface hardness. The result is a sudden increase in the contact size and a decrease in contact resistance. Contact softening in MEMS switches has already been demonstrated. ${ }^{7}$ This paper shows that the voltage required to soften ballistic contacts rises as the contact size decreases. A

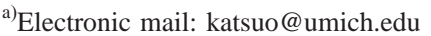

simple model explaining the phenomenon agrees well with the experimental data.

All experiments were performed using micromachined gold switches, such as the one shown in Fig. 1. The switch consists of a gold beam 3.1- $\mu \mathrm{m}$ thick, suspended 1.54- $\mu \mathrm{m}$ above a gold contact electrode. Voltage applied to the actuation electrodes produces electrostatic force, which pulls the beam down until a dimple protruding from the bottom of the beam contacts the lower electrode. The contact resistance is then measured using the four-point-probe technique, as shown in the figure. The MEMS chip is mounted on a thermal stage in an environmental chamber, allowing control of ambient temperature and pressure. All experiments were performed in vacuum of 4-5 mTorr and with sufficient actuation voltage for a contact force of $\sim 30 \mu \mathrm{N}$. This force was estimated by comparing our electrostatic/mechanical model with the measured actuation voltage. The vacuum level is sufficient to reduce the effects of moisture, which tends to cause adhesion.

Testing of the switch demonstrates that the contact resistance typically rises slowly as the switch is cycled on and off. This phenomenon has been observed in silver contacts, ${ }^{8}$ but it has never been adequately explained. It may be related to hardening of the contact due to contact necking during opening of the switch. ${ }^{3,9}$ Yield stress of gold has also been found to increase significantly as contact size decreases, ${ }^{10,11}$ which could contribute further to the contact resistance increase. The observed resistance increase could also be due to the growth of insulating hydrocarbon films on the gold surface. ${ }^{4,12}$ However, the large currents used in these experi-

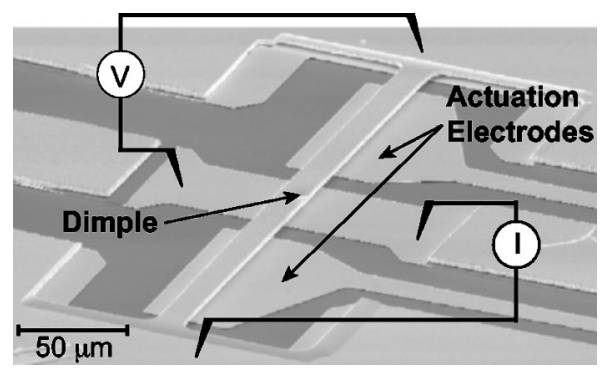

FIG. 1. Scanning electron microscopy (SEM) of a micromachined switch and the circuit used for resistance measurement. 


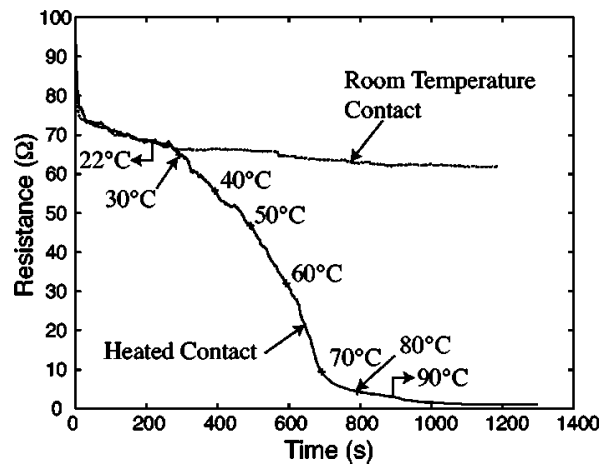

FIG. 2. Contact resistance measured over time for a heated and nonheated contact.

ments (as high as $200 \mathrm{~mA}$ ) and the linearity of the voltagecurrent relationship up to the softening voltage indicate the occurrence of metal-to-metal ohmic contact, though the size of the contact could be limited by the growth of a hydrocarbon film. Under this hypothesis, contact cycling would cause a hole in the hydrocarbon film to shrink, reducing the size of the metal-to-metal contact and increasing the contact resistance. However, regardless of the source of the observed resistance increase, it allows experiments to be performed over a wide range of contact sizes by cycling the contact until the desired contact resistance is reached.

Figure 2 shows that resistance decrease is related to the heating of the contact. Here, two contacts were measured, one heated and the other at room temperature. For the heated contact, the thermal stage was used to increase the temperature from 22 to $90{ }^{\circ} \mathrm{C}$ while measuring contact resistance. The room-temperature contact shows a small decrease in contact resistance with time due to creep, which has been previously observed for gold contacts. ${ }^{13}$ The heated contact shows enhanced contact creep almost as soon as heating begins $\left(\sim 28{ }^{\circ} \mathrm{C}\right)$, with the maximum resistance decrease occurring at $68{ }^{\circ} \mathrm{C}$.

Sample voltage-current curves for three contacts with different contact resistance values measured in a roomtemperature ambient $\left(22^{\circ} \mathrm{C}\right)$ are shown in Fig. 3. Each curve begins linearly, followed by a rapid decrease in contact resistance. This contact softening takes place at a higher voltage as the resistance increases. Therefore, the voltage required to heat the smaller contacts to the softening temperature increases with smaller contact size, contrary to Eq. (1). This is because in contact spots much smaller than the meanfree path electrons move ballistically across the contact spot,

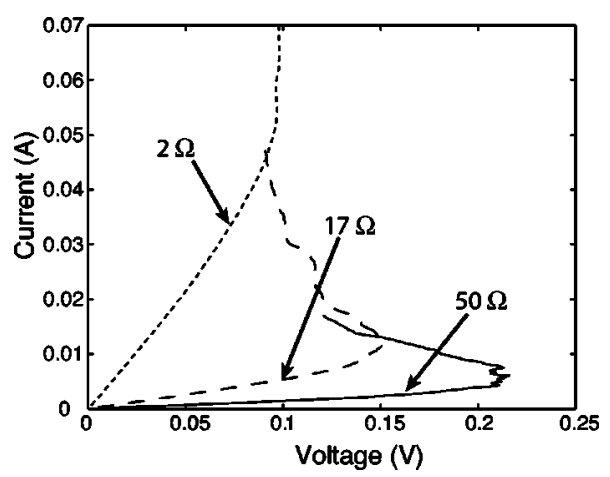

FIG. 3. Voltage-current curves showing softening. The resistance corresponding to the linear part of each curve is marked.

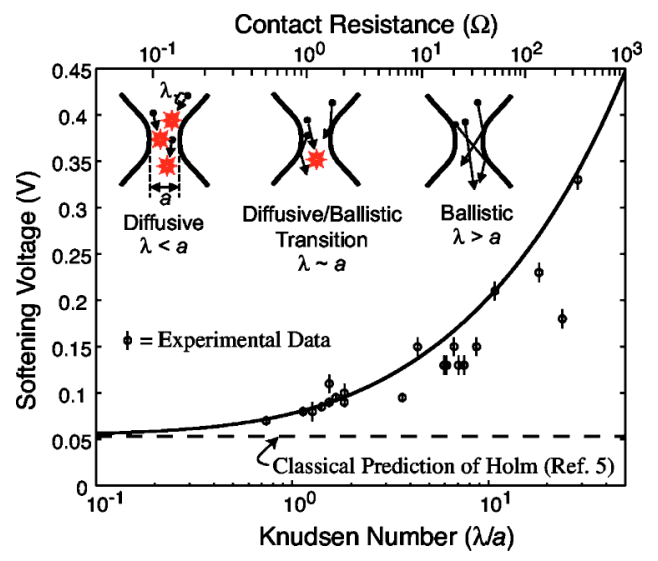

FIG. 4. Measured values of softening voltage required for contacts in ballistic-diffusive transition. Measurements are compared to the current model (solid line) and the classical model (dashed line).

so that they are unlikely to be scattered by lattice vibration quanta, phonons (for an illustration, see the inset to Fig. 4). As a result, the contact is not heated by the passage of current. The contact resistances shown in Fig. 3 are caused by contact spots in the transition between diffusive and ballistic transport; as a result, increasingly larger voltages are required for heating.

Contact resistance in the transition regime between ballistic and diffusive transport is given by

$$
R=R_{S}+\gamma(\lambda / a) R_{M},
$$

where $R$ is the total contact resistance, $R_{S}=4 \rho \lambda / 3 \pi a^{2}$ is the Sharvin resistance, or resistance due to ballistic transport, $R_{M}=\rho / 2 a$ is the Maxwell resistance, or resistance due to diffusive transport, and $\gamma(\lambda / a)$ is an interpolation function. ${ }^{14}$ $\lambda$ is the electron mean-free path, and $a$ is the radius of the contact spot.

Using Eq. (2) an approximate model for heating of transition-region contact spots can be derived. Assuming that the voltage drop $V$ in the contact can also be broken into Sharvin and Maxwell components as $V=V_{S}+V_{M}=I\left(R_{S}\right.$ $\left.+\gamma R_{M}\right)$, the contact heat generation can be expressed as $I V_{M}=I^{2} \gamma R_{M}$. Following the derivation of Holm then results in $^{5}$

$$
\frac{\gamma R_{M}}{R} V^{2}=8 \int_{T_{0}}^{T_{a}} \rho_{e} \kappa_{e} d T,
$$

where $\rho_{e}$ and $\kappa_{e}$ represent the effective electrical resistivity and thermal conductivity for transition-region electron transport. Comparison with Eq. (1) shows that the only changes are the introduction of the ratio on the left-hand side and the use of effective properties on the right-hand side. Further, since the Wiedemann-Franz law has been shown to apply even at atomistic length scales, ${ }^{15}$ Eq. (3) can be further simplified for metal contacts to

$$
\frac{\gamma R_{M}}{R} V^{2}=8 \int_{T_{0}}^{T_{a}} L T d T=4 L\left(T_{a}^{2}-T_{0}^{2}\right) .
$$

Equation (4) describes the heating in a single contact spot; however, different spots within a given area are expected to have different sizes. Therefore, further prediction of contact spot size distribution is necessary to fully model 
contact heating. However, it is simpler to consider the temperature of the largest contact spot, or the maximum contact temperature. Fractal modeling gives ${ }^{16}$

$$
A_{T}=\frac{D-1}{3-D} A_{L}
$$

where $A_{T}$ is the total contact area, $D$ is the fractal dimension (a parameter between 2 and 3), and $A_{L}$ is the area of the largest contact spot. AFM imaging of the sputtered gold surfaces used for the experiments here showed that $D$ for this case is nearly 2 , so that $A_{T}$ is approximately $A_{L}$ (meaning that all other contact spots are much smaller than the largest spot). In this case, the contact can be considered as a singleasperity contact, allowing Eq. (4) to be used directly.

The softening voltage for several measured contacts is shown in Fig. 4 compared to the predictions of the classical model of Eq. (1) and the current model of Eq. (4). For this plot, the mean-free path was $38 \mathrm{~nm},{ }^{17}$ and $T_{a}$, the softening temperature, was $68^{\circ} \mathrm{C}$ (as measured from Fig. 2). Electrical resistivity was measured as $3.6 \times 10^{-8} \Omega \mathrm{m}$. The figure inset illustrates electron transport in the diffusive, transitional, and ballistic regimes. The model describes the data trends very well, though it tends toward overprediction of softening voltage for larger resistance. This may be explained by the reduction in gold-melting temperature for particles smaller than $10 \mathrm{~nm}$ (above a Knudsen number of $\sim 4$ ). ${ }^{18}$ It follows that softening temperature may also decrease for small contact spots, leading to overprediction of softening voltage at elevated resistance. The good agreement in Fig. 4 develops confidence in the model of Eq. (4).

In conclusion, we have demonstrated that, because their contact force is typically small enough to cause the effective contact size to be comparable to the electron mean-free path, the ballistic transport of electrons governs asperity heating in MEMS switches. The rapid decrease of contact resistance with contact voltage was shown as a clear indication of contact softening. This contact softening process may provide a means for in situ repair of metal-metal contact MEMS switches.

This work is supported under a National Defense Science and Engineering Graduate Fellowship and by the NSF under CAREER Award and Grant No. ECS-0330963.

${ }^{1}$ R. S. Timsit, IEEE Trans. Compon., Hybrids, Manuf. Technol. 6, 115 (1983).

${ }^{2}$ D. Erts, H. Olin, L. Ryen, E. Olsson, and A. Tholen, Phys. Rev. B 61, 12725 (2000).

${ }^{3}$ D. Erts, A. Lohmus, R. Lohmus, H. Olin, A. V. Pokropivny, L. Ryen, and K. Svensson, Appl. Surf. Sci. 188, 460 (2002).

${ }^{4}$ J. W. Tringe, T. A. Uhlman, A. C. Oliver, and J. E. Houston, J. Appl. Phys. 93, 4661 (2003).

${ }^{5}$ R. Holm, Electric Contacts-Theory and Applications (Springer-Verlag, Berlin, 1967).

${ }^{6}$ T. N. Todorov, Philos. Mag. B 77, 965 (1998).

${ }^{7}$ B. D. Jensen, L. W. Chow, R. F. Webbink, K. Saitou, J. L. Volakis, and K. Kurabayashi, in Proceedings of the 17th IEEE International Conference on Micro Electro Mech. Systems (IEEE, Piscataway, NJ, 2004), pp. 137140.

${ }^{8}$ M. Hasegawa, T. Yamamoto, and K. Sawa, IEEE Trans. Compon., Hybrids, Manuf. Technol. 15, 177 (1992).

${ }^{9}$ J. I. Pascual, J. Méndez, J. Gómez-Herrero, A. M. Baró, N. García, and V. T. Binh, Phys. Rev. Lett. 71, 1852 (1993).

${ }^{10}$ U. Landman, Solid State Commun. 107, 693 (1998).

${ }^{11}$ S. Tanimori and S. Shimamura, J. Phys. Soc. Jpn. 71, 797 (2002).

${ }^{12}$ S. Mori, Y. Shitara, K. Takahashi, and M. Hayashi, in Proceedings of the 43rd IEEE Holm Conference on Electrical Contacts (IEEE, Piscataway, NJ, 1997), pp. 183-189.

${ }^{13}$ R. E. Cuthrell and D. W. Tipping, J. Appl. Phys. 44, 4360 (1973).

${ }^{14}$ B. Nikolić and P. B. Allen, Phys. Rev. B 60, 3963 (1999).

${ }^{15}$ S. Ciraci, A. Buldum, and I. P. Batra, J. Phys.: Condens. Matter 13, R537 (2001).

${ }^{16}$ W. Yan and K. Komvopoulos, J. Appl. Phys. 84, 3617 (1998).

${ }^{17}$ N. W. Ashcroft and N. D. Mermin, Solid State Physics, 1st ed. (Holt, Rinehart and Winston, New York, 1976).

${ }^{18}$ P. Buffat and J.-P. Borel, Phys. Rev. A 13, 2287 (1976). 